\title{
A phantom gallbladder on endoscopic retrograde cholangiopancreatography
}

\author{
Jinhong Xing, Jeremy Rochester, Caroline K Messer, Bruce P Reiter, Mark A Korsten
}

Jinhong Xing, Jeremy Rochester, Caroline K Messer, Bruce P Reiter, Mark A Korsten, Gastroenterology and Radiology Programs, James J. Peters VA Medical Center, Bronx, NY 10468, Montefiore Medical Center, Bronx, NY, Mount Sinai School of Medicine, New York, NY and Albert Einstein College of Medicine, Bronx, New York 10468, United States

Correspondence to: Mark A Korsten, MD, James J Peters VA Medical Center, 130 W. Kingsbridge Road, Bronx, New York 10468, United States. korsten.mark@med.va.gov

Telephone: +1-718-5849000-6753 Fax: +1-718-5791635

Received: April 20, 2007

Revised: September 6, 2007

\begin{abstract}
Various complications have been related to laparoscopic cholecystectomy but most occur shortly after the procedure. In this report, we present a case with very late complications in which an abscess developed within the gallbladder fossa 6 years after laparoscopic cholecystectomy. The abscess resolved after treatment with CT-guided extrahepatic aspiration. However, 4 years later, an endoscopic retrograde cholangiopancreatography (ERCP) performed for choledocholithiasis demonstrated a "gallbladder" which communicated with the common bile duct via a patent cystic duct. This unique case indicates that a cystic duct stump may communicate with the gallbladder fossa many years following cholecystectomy.
\end{abstract}

\section{(c) 2007 WJG. All rights reserved.}

Key words: Laparoscopic cholecystectomy; Complication; Abscess; Gallbladder; Endoscopic retrograde cholangiopancreatography

Xing J, Rochester J, Messer CK, Reiter BP, Korsten MA. A phantom gallbladder on endoscopic retrograde cholangiopancreatography. World J Gastroenterol 2007; 13(46): 6274-6276

http://www.wjgnet.com/1007-9327/13/6274.asp

\section{INTRODUCTION}

Laparoscopic cholecystectomy has been widely accepted as a preferred option for the management of patients with cholelithiasis. Complications such as biliary injuries ${ }^{[1,2]}$ intestinal ischemia ${ }^{[3]}$ and biliary-colonic fistula ${ }^{[4]}$ have been reported, but most occur shortly after surgery. In this report, we present a case with very late complications in which an abscess developed within the gallbladder fossa 6 years after laparoscopic cholecystectomy. Four years later, despite the clinical certainty that it had been removed 10 years previously, a "gallbladder" communicating with the common bile duct via a cystic duct was demonstrated by endoscopic retrograde cholangiopancreatography (ERCP).

\section{CASE REPORT}

A 73-year-old male presented with complaints of right upper quadrant discomfort, fatty food intolerance and bloating. He eventually underwent an elective laparoscopic cholecystectomy in early October 1996. A J-P drainage tube was placed during the operation. The drainage was initially dark bloody, then became bilious, but gradually became clear over the next several days. The patient was discharged home after 1 wk. A follow-up abdominal CT scan was performed in May 1997, six months after the procedure. There were no fluid collections in the gallbladder fossa or peritoneal cavity.

The patient did well until early October 2002, six years after cholecystectomy, when he presented with complaints of fever, chills and mild pain in the right upper quadrant. Sonographic examination of the abdomen showed a normal sized liver with non-dilated intrahepatic ducts and common bile duct. Of note, a gall bladder-like structure was seen in the gallbladder fossa. An abdominal CT confirmed the presence of a $4 \mathrm{~cm}$ encapsulated fluid collection in the gallbladder fossa (Figure 1). A subsequent HIDA scan showed prompt excretion of the radiotracer into the small bowel with no evidence of biliary leak. A CT-guided transhepatic intra-abdominal aspiration was then performed (Figure 2). Turbid-appearing fluid was readily aspirated from the extrahepatic fluid collection. Gram stain of the aspirated fluids showed the presence of many white blood cells. Cultures were positive for moderate Enterobacter SP in the aspirates. The persistent low-grade fever subsided shortly after the CT-guided aspiration, and the patient was discharged home with no abdominal complaints. In August 2004 (22 mo after aspiration), an abdominal CT scan was repeated and no gallbladder fossa fluid collection was observed.

In May 2006, the patient was again admitted with the sudden onset of abdominal pain, icterus and fever. A CT scan showed a dilated common bile duct. MRCP showed intra- and extrahepatic biliary 


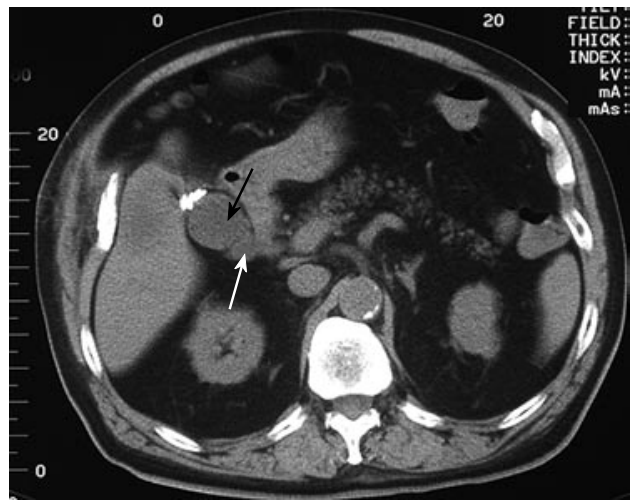

Figure 1 Abdominal CT scan six years after laparoscopic cholecystectomy. A4 cm encapsulated fluid collection in the gallbladder fossa (black arrow) with adjacent "cystic duct-like" collections was observed (white arrow).

dilatation as well as a round, low signal filling defect within the distal common bile duct. During ERCP, the cholangiogram revealed a mildly dilated proximal common bile duct with 2 mobile stones (Figure $3 \mathrm{~A}$ ). A gallbladder-like structure that communicated with the common bile duct was also visualized in the gallbladder fossa (Figure 3B). Sphincterotomy was performed, and numerous stone fragments were removed from the CBD. A plastic biliary endoprosthesis (10F) was placed in the distal common bile duct. The patient was discharged home without any complication or discomfort two days after the ERCP. One month later, the stent was removed and the common bile duct appeared normal. A "phantom" gallbladder image reappeared on the cholangiogram.

\section{DISCUSSION}

It is not uncommon to develop a fluid collection in the gallbladder fossa shortly after laparoscopic cholecystectomy. Kang et $\left.\right|^{[5]}$ studied 106 consecutive patients $24 \mathrm{~h}$ after laparoscopic cholecystectomy using ultrasound. They identified small fluid collections in the gallbladder fossa in $56(53.0 \%)$ patients. In another study ${ }^{[6]}$, the gallbladder fossa of 70 asymptomatic patients was sonographically examined within two weeks of laparoscopic cholecystectomy. The authors reported the presence of homogeneous echogenic structures in the gallbladder bed in $35(50 \%)$ patients, inhomogeneous structures in $25(35.7 \%)$ cases, and, cystic structures, resembling the normal gallbladder, in $6(8.5 \%)$ cases. The nature of the fluid collection varied, but included seromas, hematomas, abscesses and biloma.

In the present case, the fluid collection inside the gallbladder fossa observed 6 years after the procedure proved to be an abscess on the basis of the turbid, nonbilious, culture positive fluid retrieved during CT-guided aspiration. The abscess resolved after percutaneous transhepatic aspiration and antibiotic treatment. The most recent finding in this patient, a gallbladder-like structure inside the gallbladder fossa that directly communicated with the common bile duct, is likely related in some manner to this prior abscess. One possibility is that the previously documented abscess may have resolved by

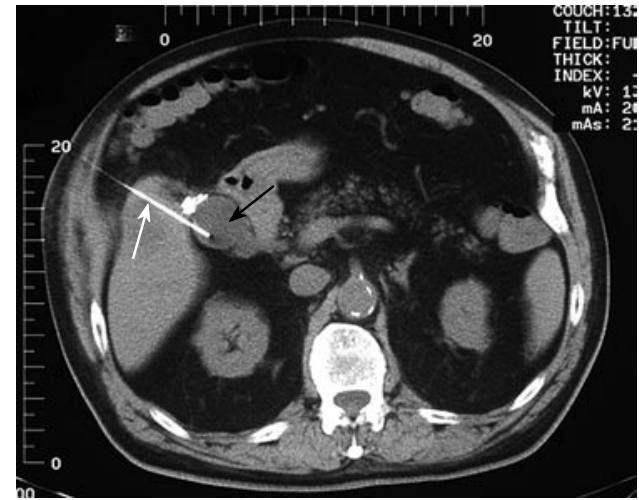

Figure 2 A CT-guided transhepatic percutaneous aspiration needle (white arrow) was seen entering the extrahepatic fluid collection (black arrow).

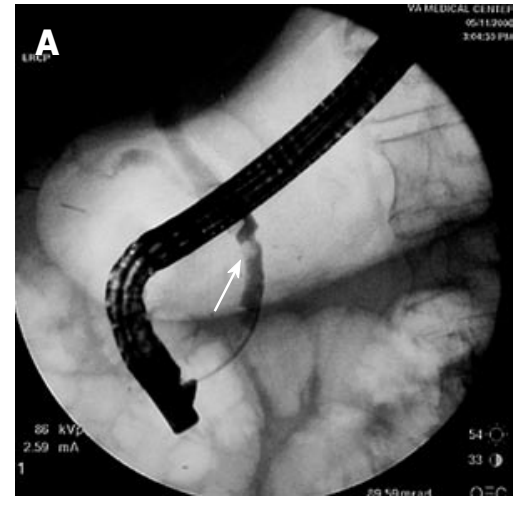

Figure 3 Calculi (white arrow) in the common bile duct were noted during the $\operatorname{ERCP}(\mathbf{A})$. In addition, a gallbladder-like structure (black arrow) communicated with the common bile duct via the cystic duct (white arrow) was also observed (B).

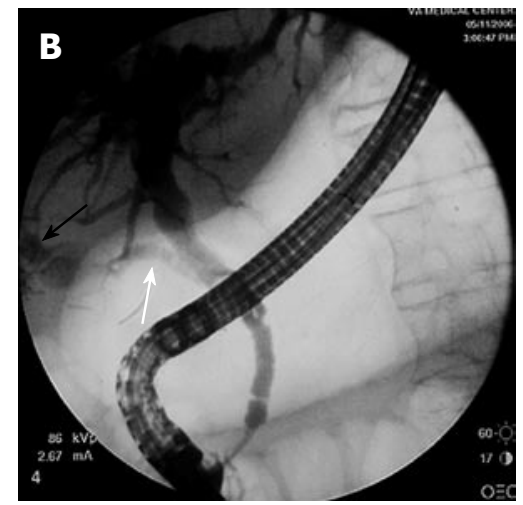

fistualizing into the cystic duct. Alternatively, increased pressure within the cystic duct as a result of biliary obstruction from choledocholithiasis may have caused rupture of the stump and its subsequent breakthrough into a residual cavity in the gallbladder fossa.

By whatever mechanism, this case demonstrates that, post-cholecystectomy, a cystic duct stump may potentially communicate with extrabiliary structures in and around the gallbladder fossa. In this respect, we believe that this is the first reported case in which a cystic structure resembling a radiologically typical "gallbladder" was seen on ERCP many years after this organ was surgical removed.

\section{REFERENCES}

1 Archer SB, Brown DW, Smith CD, Branum GD, Hunter JG Bile duct injury during laparoscopic cholecystectomy: results of a national survey. Ann Surg 2001; 234: 549-558; discussion 558-559 
2 Wright TB, Bertino RB, Bishop AF, Brady TM, Castaneda F, Berkman WA, Finnegan MF. Complications of laparoscopic cholecystectomy and their interventional radiologic management. Radiographics 1993; 13: 119-128

3 Leduc LJ, Mitchell A. Intestinal ischemia after laparoscopic cholecystectomy. JSLS 2006; 10: 236-238

4 Munene G, Graham JA, Holt RW, Johnson LB, Marshall HP Jr. Biliary-colonic fistula: a case report and literature review. Am
Surg 2006; 72: 347-350

5 Kang EH, Middleton WD, Balfe DM, Soper NJ. Laparoscopic cholecystectomy: evaluation with sonography. Radiology 1991; 181: 439-442

6 Doringer E, Forstner R, Hölbling N, Moritz E, Schmoller H. [Ultrasound image of the gallbladder fossa after cholecystectomy in the immediate postoperative period]. Ultraschall Med 1991; 12: 248-251

S- Editor Liu Y L- Editor Ma JY E- Editor Ma WH 